\title{
Incorporación de la simulación en el entrenamiento del manejo de recursos en crisis, experiencia del programa de anestesiología de la Pontificia Universidad Católica de Chile
}

\author{
Carlos Fuentes Amaya ${ }^{1}$, Marcia Corvetto Aqueveque ${ }^{1}$, Fernando Altermatt Couratier ${ }^{1}$, Alejandro Delfino Yurín ${ }^{*}$
}

\begin{abstract}
Resumen: La adecuada conducción de situaciones críticas en medicina requiere desarrollar un intrincado proceso cognitivo para generar acciones precisas con estrechos márgenes de error; sin embargo, alcanzar estos objetivos suele ser complejo y representa un desafío insalvable a la educación médica tradicional; en este contexto, la implementación del entrenamiento en simulación para el manejo de recursos en crisis (MRC) ha mostrado ser la herramienta educativa mas efectiva para la adquisición y retención de habilidades en el tiempo. Presentamos una revisión narrativa de los objetivos de aprendizaje que persigue el MRC describiendo la utilidad educativa de la simulación y el proceso de integración de esta metodología al currículo de anestesiología de la Pontificia Universidad Católica de Chile.
\end{abstract}

Palabras clave: educación; simulación; crisis; interrogatorio; anestesiología.

Abstract: An actuated management of critical situations in medicine requires an intricate cognitive process to generate "on target" actions under narrow margins of error; however, the achievement of this goal is often complex and represents an absolute challenge to traditional medical education. In this context, the use of simulation for crisis resource management (CRM) has proven to be the most effective teaching approach for the acquisition and retention of skills over time. We present a narrative review of the learning objectives pursued by CRM, describing the utility and integration process of this technique into the curriculum of anesthesiology at Pontificia Universidad Católica de Chile.

Keywords: Medical education; simulation; crisis resource management; debriefing; anesthesiology.

Fecha de envío: 11 de octubre de 2016 - Fecha de aceptación: 3 de marzo de 2017

\section{Introducción}

En nuestro medio, la magnitud del error médico sigue siendo desconocida debido a su falta de reconocimiento y registro; sin embargo, en EE. UU., este problema es considerado actualmente como la tercera causa de morbimortalidad intrahospitalaria, poniendo de manifiesto la necesidad imperativa de generar y mantener estrategias que permitan mejorar la calidad de la atención en salud (Makary et al.; 2016).

Si bien los mecanismos promotores de error surgen de las limitaciones psicológicas, fisiológicas y cognitivas propias del ser humano, también dependen de factores modificables, como agotamiento físico, falta de habilidades comunicacionales, mal manejo del estrés, interpretación equivocada de información y fallas de planificación, los que de no ser corregidos en forma oportuna, aumentan el riesgo de manejar inadecuadamente situaciones críticas no anticipadas (Gaba,1989, Gaba \& Howard, 2002; Khon et al., 1999).

Por su parte, el entrenamiento en simulación para el manejo de recursos en crisis ha mostrado que optimiza los procesos cognitivos requeridos para la toma de decisiones complejas y fomentar el trabajo en equipo; ello ha aumento progresivamente el interés por integrarlo formalmente al currículo de diferentes programas de formación médica (Chiu et al., 2016, Issenberg et al., 2005).

El propósito de este manuscrito es reforzar el concepto del MRC, destacar la importancia de su entrenamiento mediante la simulación, y describir la forma en la que se implementó esta metodología dentro del currículo de anestesiología de la Pontifica Universidad Católica de Chile.

(1) División de Anestesiología, Escuela de Medicina, Pontificia Universidad Católica de Chile

*Autor decorrespondencia: aedelfin@med.puc.cl 
Fuentes et al.

\section{Definición:}

El MRC es un concepto derivado de la aviación y adaptado a la medicina a través de la anestesiología (Gaba, 1992; Gaba et al.,1994); persigue reconocer, coordinary utilizar, de la mejor forma posible, todos los recursos humanos y técnicos disponibles para mejorar la seguridad del paciente antes de enfrentar posibles situaciones caóticas; así mismo, facilita la detección oportuna de factores promotores de error durante las mismas y la generación de acciones encaminadas a minimizar su impacto (Howard et al., 1992; Gaba et al., 2001; Gaba, 2015; Helmreich, 2000).

Debido a la baja tasa de ocurrencia de eventos críticos y la subsecuente complejidad que ofrece la generación de competencias para su adecuado enfrentamiento, Gaba y colaboradores propusieron el desarrollo de escenarios críticos simulados para el entrenamiento en MRC constituyendo uno de los mayores aportes a la educación médica de los últimos tiempos (Alison et al., 2013, Gaba et al., 2001).

\section{Objetivos de aprendizaje:}

Si bien esta modalidad pedagógica busca fomentar el desarrollo sincrónico de múltiples habilidades (tabla 1), también permite que el docente se enfoque selectivamente en algún área dirigida al perfil de competencias requerido para cada situación particular (Gaba, 2001). Para nosotros, las características más relevantes que sustentan la estructura del MRC y que se abordan de forma reiterativa durante los escenarios críticos son:

a) Comunicación efectiva: las habilidades comunicativas suelen ser complejas de adquirir y mantener aún en situaciones controladas; si se extrapola esta situación a un escenario crítico, es esperable encontrar que lo verbalizado no siempre sea entendido y traducido en una acción concreta (Miller, 2009; Gaba 2015); por su parte, el entrenamiento en simulación permite fomentar el liderazgo, promover el orden, y generar herramientas para garantizar comprensión y continuidad en las acciones de todos los miembros del equipo bajo un modelo de comunicación efectiva.

b) Anticipación y planificación: dentro del desarrollo de un escenario de crisis, se motiva al alumno a estar alerta y suponer que siempre algo puede ir mal; de esta forma, se pretende que disponga habitualmente de planes estratégicos y recursos suficientes para afrontar idóneamente situaciones complejas no anticipadas (Gaba, 2015).

c) Prevención y manejo adecuado de los errores de fijación: este fallo cognitivo implica la incapacidad de revisar o cambiar un plan pese a que hay evidencia suficiente para hacerlo (Fioratu \& Glavin, 2010; Gaba,1992). Está determinado por la dificultad que ofrece el enfrentar situaciones en las que las pistas preliminares no definen completamente una característica condicionando al sujeto a asumir que la suposición inicial es indudablemente correcta (Gaba, 1992; Schwid \& O'Donnell, 1992). Un ejemplo concreto es interpretar insistentemente una capnografía y saturometría bajas como producto de broncoespasmo o tromboembolismo pulmonar sin reconocer oportunamente y como causa más probable una intubación esofágica. Pese a que este problema es inherente a todo ser humano y es potencialmente corregible, toma gran relevancia cuando persiste dentro de una situación crítica.

El análisis retrospectivo de las acciones tomadas durante un caso simulado permite fomentar la capacidad de autocrítica considerando ampliamente otros puntos de vista; de esta forma se construyen los principales mecanismos modificadores de la fijación (Gaba, 1992; Fanning \& Gaba, 2007).

d) Evaluación repetida de la situación: en virtud de que las crisis médicas no son eventos estáticos, se espera que el alumno se replantee constantemente los diagnósticos y las conductas terapéuticas tomadas manteniéndose siempre alerta ante eventuales cambios; la metodología de trabajo en simulación enfatiza constantemente la importancia de la realización de pausas para preguntarse si las cosas van bien y de esta forma, cambiar lo que sistemáticamente pueda conducir a error (Miller, 2009; Gaba, 2015).

e) Conocimiento del entorno: se espera que el entrenado tenga claridad absoluta de su entorno de trabajo, lo que implica conocer con quién trabaja, qué características de las instalaciones físicas son relevantes, a quién debe llamar para solicitar ayuda, cómo hacerlo, qué equipos están disponibles, dónde puede obtenerlos, cómo se usan, cómo debe revisarlos y qué ayudas cognitivas puede incorporar al ámbito laboral. (Miller, 2009; Gaba, 2015).

\section{Tabla 1: Puntos clave en el manejo de recursos en crisis}

1. Conozca el entorno

2. Anticípese y planifique

3. Solicite ayuda precozmente

4. Promueva el liderazgo y la adherencia al mismo

5. Distribuya la carga laboral: asigne funciones

6. Movilice todos los recursos disponibles

7. Comuníquese efectivamente: Hable!

8. Use toda la información disponible

9. Prevenga y maneje adecuadamente los errores de fijación

10. Realice doble lista de chequeo: nunca de algo por hecho

11. Use ayudas cognitivas: algoritmos, internet

12. Re evalúe repetidamente la situación: 10 segundos por 10 minutos

13. Establezca un buen equipo de trabajo

14. Fije su atención de forma racional

15. Organice las prioridades de forma dinámica

Adaptado de Rall M, Gaba DM: Human Performance and Patient Safety, in Miller 7th edition 2005 


\section{Fundamentos pedagógicos para el uso de la simulación en MRC}

Tradicionalmente, en medicina se usa el análisis retrospectivo de casos clínicos para identificar fuentes de error, revisar temas y fijar estrategias para mejorar la toma de decisiones clínicas; sin embargo, esta aproximación se contrapone al principio de anticipación y ofrece sesgos de selección por cuanto considera solo aquellas instancias percibidas como complejas o interesantes; por otra parte, recuerda lo ocurrido a partir de registros que pueden no ser completamente fiables y no siempre integra activamente a otros miembros del equipo médico (Gaba,1992; Schwid, 1992); como resultado, la información cognitiva generada es procesada principalmente en la memoria de trabajo por lo que es esperable que lo aprendido se olvide con mayor rapidez (Gaba, 1992).

Por el contrario, la simulación actúa como mecanismo de "neuropriming" mejorando los procesos de adquisición de habilidades más allá del sentido común (Issenberg et al., 2005); por medio de la sensibilización neo cortical, genera una impronta psicomotora ligada a un recuerdo emotivo que magnifica el aprendizaje, mejora la memoria y optimiza la retención de competencias en el tiempo (Catania \& Dell'Aqcuaa, 2013). Lo anterior es aplicable a los escenarios de crisis en los que se reproducen situaciones de diversos niveles de dificultad donde todos los miembros de un equipo toman acciones "bedside" y en tiempo real para posteriormente durante el interrogatorio (debriefing), identificar mecanismos promotores de error y usarlos como pauta para construir conocimiento (Dieckmann et al., 2009; Gaba et al., 2010, Paige et al., 2015); si bien su propósito es mejorar fundamentalmente comportamientos y no habilidades técnicas, ambas instancias no son mutuamente excluyentes y se abordan de forma balanceada durante el desarrollo y análisis pedagógico de cada caso (Alison et al., 2013).

\section{Proceso de integración curricular en anestesiología y selección de escenarios:}

Debido a que la práctica anestesiológica ofrece una mayor exposición a situaciones complejas no anticipadas en las que aumenta la probabilidad de errar, se hace necesario implementar y mantener estrategias periódicas de entrenamiento protegido en pro de mejorar la seguridad del paciente (Gaba et al., 2001). Esta observación justifica por si sola la inclusión de la simulación como parte de las actividades pedagógicas de cualquier programa de formación de anestesiólogos; sin embargo, el proceso de integración curricular de esta metodología es exigente y requiere de una adecuada justificación académica y consenso docente; es por esto que, al momento de tomar decisiones políticas para definir su ingreso formal al programa de anestesiología de la Pontificia Universidad Católica de Chile, otros factores tuvieron que ser considerados:

\section{a) Perfil profesional deseado por la Universidad:}

El programa de anestesiología de la Pontificia Universidad Católica de Chile pretende formar profesionales con conocimientos teóricos y destrezas necesarias para desempeñarse en cualquier servicio de anestesiología del país o incorporarse a programas de especialización en áreas de mayor complejidad; desde etapas precoces del proceso educativo, busca que el residente sea autónomo, líder de opinión, artífice de cambios y promotor de armonía con otros miembros del equipo de trabajo. Por su parte, la dirección de postgrado de la Pontificia Universidad Católica de Chile ha trabajado en la introducción del marco de competencias canadienses CanMeds a los programas de especialización médica con el objetivo de generar siete grandes características: ser experto, comunicador, colaborador, promotor de la salud, administrador, estudioso y profesional. A la luz de estas directrices, el enfoque pedagógico que ofrece la simulación para el aprendizaje de MRC hace más fácil justificar cualquier política de modificación curricular requerida para su implementación.

\section{b) Disponibilidad de medios para acometer esta tarea:}

La simulación es una instancia intermedia, que no pretende suplantar la realidad pero que requiere de un adecuado espacio físico e insumos materiales para garantizar su efectividad académica; por esta razón es frecuente que sea percibida como una herramienta de alto costo dificultando su adopción; sin embargo, existe evidencia suficiente para considerar que no es imperativo invertir en tecnología extremadamente avanzada para producir resultados favorables; basta con que el modelo físico y el diseño de caso reproduzcan razonablemente una situación clínica para que se cumplan los objetivos de aprendizaje (Cook et al., 2011; Salas et al., 2008); de cualquier forma, es imprescindible contar con un equipo docente altamente calificado para diseñar, modificar, montar y evaluar cada uno de los escenarios bajo la metodología apropiada (Salas et al., 2008).

En nuestro caso, disponemos de un laboratorio de más de 100 $\mathrm{m}^{2} \mathrm{y}$ de un cuerpo docente conformado por tres anestesiólogos formados en simulación y conducción del interrogatorio final; uno de ellos es además el director del centro de simulación de la escuela de Medicina. Todas estas condiciones facilitan no solo la implementación de los escenarios críticos a la plataforma curricular, sino también la generación de proyectos de investigación y la interacción constante con otros profesionales de la salud.

\section{c) Análisis del currículo de anestesiología}

La integración de la simulación a nuestra residencia partió con un riguroso mapeo curricular que determinó la forma en la que las competencias definidas por nuestro programa estaban siendo 
enseñadas, si es que esto ocurría, y los métodos que estaban siendo empleados para su evaluación. Al final del proceso, se objetivaron algunos vacíos curriculares susceptibles de ser resueltos mediante una estrategia pedagógica integrativa; con base en ello y bajo concordancia plena con los lineamientos propuestos por el CanMeds y el perfil profesional buscado por nuestra universidad, se construyó una lista preliminar de 9 escenarios críticos propicios para ser enseñados mediante simulación:

- Paro cardio-respiratorio

- Vía aérea difícil

- Anafilaxia

- Arritmia

- Hipertermia Maligna

- Intoxicación por anestésicos locales

- Hemorragia obstétrica

- Politraumatismo

- Shock hemorrágico

Si bien todas estas situaciones implican tener un margen mínimo de conocimientos teóricos sobre la patología o condición específica a tratar, su objetivo principal es construir habilidades en comunicación, trabajo en equipo y administración de recursos donde la simulación ha mostrado ventajas comparativas en relación con otros métodos tradicionales de enseñanza (Chiu et al., 2016, Fraser et al., 2016; Levine \& Shorten G, 2016).

\section{d) Análisis y búsqueda en la literatura}

Con el objetivo de conocer cuáles habían sido los escenarios más usados en educación médica, se efectuó una búsqueda en la literatura usando las bases de datos Medline, Centro de Información y Recursos Educacionales (ERIC), Índice Educacional Británico (BREI) e Índice Educacional Australiano (AVEI). Los términos de búsqueda empleados fueron: simulación, anestesia, entrenamiento en crisis, educación, manejo de crisis, competencias clínicas, validez y confiabilidad. Se incluyeron únicamente artículos publicados en inglés entre enero de 1965 y marzo de 2014 estableciendo su relevancia mediante la información contenida en el título y/o resumen; paralelamente, se condujo una selectiva búsqueda manual basada en referencias extraídas de artículos de revisión descartando aquellos efectuados con animales o lejanos al área médica.

Los escenarios resultantes de este proceso fueron absolutamente concordantes con los generados por el mapeo curricular; además, surgieron dos nuevas propuestas de trabajo: falla de máquina de anestesia o equipos y entrenamiento en error de fármacos.

\section{e) Consenso de expertos}

Para validar los resultados de los dos procesos anteriores, se efectuó una encuesta de consenso entre los 10 anestesiólogos a cargo de las diversas rotaciones que constituyen nuestra residencia. Mediante una escala de Likert de 4 puntos, donde 1 significaba no relevante y 4 muy relevante, cada anestesiólogo manifestó su postura con respecto a la importancia de incorporar estos escenarios al proceso de formación de residentes; adicionalmente, les fue permitido señalar algún otro escenario que no hubiese sido considerado previamente y que fuera potencialmente relevante.

Para el análisis de la información obtenida, las cuatro categorías ordinales fueron resumidas en dos categorías dicotómicas: "no relevante" y "relevancia leve" en una, y "relevante" y "muy relevante" en otra; posteriormente, se calculó el índice de validez de contenido (CVI) y el coeficiente Kappa con el fin de eliminar el efecto del acuerdo por azar considerando que solo aquellos escenarios con valores de CVI y coeficiente kappa mayores o iguales a 0,78 y 0,6 respectivamente, deberían ser incluidos en el listado definitivo.

Como resultado de este proceso, se recibieron 10 encuestas de consenso representando el $100 \%$ de los datos; los valores de CVI y coeficiente kappa variaron entre 0,8 y 1 y 0,9 y 1 respectivamente y el $80 \%$ de los encuestados sugirió la incorporación de un escenario adicional de paciente poli traumatizado; de esta forma, se construyó un portafolio final con 12 escenarios críticos simulados a incluir dentro del currículo de anestesiología de nuestra universidad (tabla 2).

Tabla 2: Escenarios simulados introducidos en el currículo de anestesiología

\begin{tabular}{|ll|}
\hline 1. & Paro cardio-respiratorio \\
\hline 2. & Vía aérea difícil \\
\hline 3. & Anafilaxia \\
\hline 4. & Arritmia \\
\hline 5. & Hipertermia Maligna \\
\hline 6. & Intoxicación por anestésicos locales \\
\hline 7. & Hemorragia obstétrica \\
\hline 8. & Politraumatismo \\
\hline 9. & Shock hemorrágico \\
\hline 10. & Falla de máquina de anestesia o equipos \\
\hline 11. & Error de fármacos \\
\hline 12. & Paciente politraumatizado \\
\hline
\end{tabular}




\section{Implementación práctica de la simulación en el programa de anestesia}

Desde julio de 2014, el programa descrito se incorporó formalmente al currículo de anestesiología de la Pontificia Universidad Católica de Chile; durante el proceso, debieron ser salvados dos inconvenientes de gran relevancia:

a) Reticencia de algunos de los docentes de planta de la División de Anestesiología

Un gran desafío inicial resultó ser la propia resistencia de algunos de los anestesiólogos de planta a la implementación de esta innovación metodológica; frases como "no veo la utilidad de esto" o"yo no aprendí así y soy buen anestesiólogo" fueron recurrentes entre varios de los integrantes del equipo. Para sortear este difícil escollo, se hizo necesario socializar el programa entre todos los integrantes de la división y permitirles observar la demostración empírica de los efectos educativos del programa a lo largo del tiempo. Al cabo de seis meses, se fue generando espontáneamente el reconocimiento docente, y los docentes fueron señalando en consenso que el manejo y comportamiento de los residentes ante situaciones complejas era sustancialmente mejor a lo evidenciado previo al uso de la simulación. En la actualidad, ya no existen cuestionamientos sobre esta herramienta pedagógica y se trabaja activamente para su adecuado desarrollo y éxito.

\section{b) Dificultades logísticas}

El programa de Anestesiología se desarrolla en diversos centros asistenciales distribuidos en diversas comunas de Santiago, siendo su centro neurálgico el Hospital Clínico de la Pontificia Universidad Católica de Chile, lugar donde, además, se encuentra ubicado el Centro de Simulación. Las actividades de los residentes en dichos campos clínicos varían sustancialmente, y van desde ser sujetos colaboradores para el proceso de atención de pacientes (sin ser indispensable su presencia constante) a ser actores imprescindibles para el desarrollo de las labores asistenciales. Dada esta diversa realidad, el lograr la asistencia de todos los residentes a esta actividad fue inicialmente complejo, particularmente por la falta de colaboración de algunos campos clínicos y la falta de disposición para el traslado por parte de los propios alumnos. Para superar este inconveniente, clave resultó el que esta instancia fuera incorporada en el currículo como actividad de carácter obligatorio, otorgándole tiempo protegido dentro de la jornada laboral habitual de la residencia. Con esta definición de carácter político, se aseguró la asistencia de todos los residentes, debiendo otorgar todos los campos clínicos, las respectivas facilidades para su concurrencia.
Finalmente, desde el punto de vista de la evaluación, el programa de anestesia de la Pontificia Universidad Católica de Chile se encuentra en una fase de migración, desde un sistema tradicional hacia la evaluación por competencias sugerida por CanMeds. Por lo pronto, asumimos que el cursar el portafolio de simulación junto a la exposición clínica propia de nuestro programa de formación de anestesiología, ha generado en los residentes las competencias deseadas. Esperamos que en el corto plazo seamos capaces de demostrar con evidencia sólida que efectivamente esto es así.

\section{Conclusiones}

El entrenamiento en simulación para el MRC es un pilar fundamental para el proceso de formación de especialistas en anestesiología. Para garantizar su adecuada integración curricular, es imprescindible conocer sus fundamentos teóricos y ventajas prácticas, mantener la concordancia con el perfil del profesional buscado por la universidad, realizar un exhaustivo mapeo curricular diagnóstico, revisar cuidadosamente la literatura para seleccionar los escenarios más relevantes, justificar con evidencia sus objetivos pedagógicos, buscar consenso docente y generar el recurso humano adecuado para administrar los insumos tecnológicos y pedagógicos requeridos. Por su parte, la correcta implementación y mantención de esta estrategia trae consigo la ejecución activa de proyectos de investigación y publicación de resultados que facilitan su adopción dentro de otros programas de formación de especialistas donde es cada vez más requerida (Hunang et al., 2016).

La división de Anestesiología de la Pontificia Universidad Católica de Chile mantiene el compromiso docente con esta modalidad de entrenamiento propendiendo incansablemente por innovar su enfoque en el tiempo; además, considera que en un futuro próximo será necesario trabajar en conjunto con representantes de otras facultades de medicina para contribuir al desarrollo de guías de trabajo en simulación que permitan generar un adecuado aprendizaje guiado por competencias para todos los profesionales de la salud (Miller G, 1990); solo de esta forma será posible aportar datos concretos sobre el rendimiento real de los equipos médicos y adoptar políticas concretas para mejorar la calidad de atención en salud en el país.

\section{Agradecimientos}

Este trabajo ha sido posible en parte gracias a los fondos otorgados por la Comisión Nacional de Investigación Científica y Tecnológica (CONICYT), FONDECYT № 1141159. 
Fuentes et al.

\section{Referencias}

Alison, L., van den Heuvel, C., Waring, S., Power, N., Long, A., O'Hara, T., \& Crego, J. (2013). Immersive Simulated Learning Environments for Researching Critical Incidents: A Knowledge Synthesis of the Literature and Experiences of Studying High-Risk Strategic Decision Making. Journal of Cognitive Engineering and Decision Making, 7, 255-272. http://doi.org/10.1177/1555343412468113

Catania M, Dell'Acquaa F. A revised limbic system model for memory, emotion and behaviour. Neurosci Biobehav Rev. Elsevier Ltd; 2013 Sep;37(8):1724-37.

Cook, D. A., Hatala, R., Brydges, R., Zendejas, B., Szostek, J. H., Wang, A. T., Hamstra, S. J. (2011). Technology-enhanced simulation for health professions education: a systematic review and meta-analysis. JAMA : The Journal of the American Medical Association, 306(9), 978-988. http://doi.org/10.1001/jama.2011.1234

Chiu, M., Tarshis, J., Antoniou, A. et al., (2016). Simulation-based assessment of anesthesiology residents' competence: development and implementation of the Canadian National Anesthesiology Simulation Curriculum (CanNASC). Can J Anesth/J Can Anesth (2016). doi:10.1007/s12630-016-0733-8

Dieckmann, P., Molin Friis, S., Lippert, A., \& Ostergaard, D. (2009). The art and science of debriefing in simulation: Ideal and practice. Medical Teacher, 31(7), e287-94. http://doi.org/10.1080/01421590902866218

Fanning RM, Gaba DM. The role of debriefing in simulation-based learning. Simul Healthc. 2007 Summer; 2(2):115-25. doi: 10.1097/ SIH.0b013e3180315539. PMID: 19088616 DOI: 10.1097/SIH. ob013e3180315539

Fioratou, E., Flin, R., \& Glavin, R. (2010). No simple fix for fixation errors: Cognitive processes and their clinical applications. Anaesthesia. http://doi.org/10.1111/j.1365-2044.2009.05994.x

Fraser AB, Stodel EJ, Chaput AJ. (2016). Curriculum reform for residency training: competence, change, and opportunities for leadership. Can J Anesth; 63: 875-84.

Gaba, D. M. (1989). Human error in anesthetic mishaps. International Anesthesiology Clinics. http://doi.org/10.1097/00004311198902730-00002

Gaba, D.M. (1992). Improving anesthesiologists' performance by simulating reality. Comment on: Anesthesiologists' management of simulated critical incidents. Anesthesiology. PMID: 1550272 [PubMed - indexed for MEDLINE]
Gaba, D. M., Fish, K. J., \& Howard, S. K. (1994). Crisis management in anesthesiology. Anesthesia \& Analgesia, 78 (6), 1210. Retrieved from http://www.hsls.pitt.edu/resources/databases/ovid/

Gaba, D. M., Howard, S. K., Fish, K. J., Smith, B. E., \& Sowb, Y. a. (2001), Simulation-Based Training in Anesthesia Crisis Resource Management (ACRM): A Decade of Experience. Simulation \& Gaming, 32 (2), 175-193. http://doi.org/10.1177/104687810103200206

Gaba, D. M., \& Howard, S. K. (2002). Patient safety: fatigue among clinicians and the safety of patients. The New England Journal of Medicine, 347 (16), 1249-1255. http://doi.org/10.1056/NEJMsa020846

Gaba, D.M. (2015). Crisis Management in Anesthesiology. Elsevier Saunders.

Helmreich, R. L. (2000). On error management: lessons from aviation. BMJ (Clinical Research Ed.), 320 (7237), 781-785. http://doi. org/10.1136/bmj.320.7237.781

Howard, S. K., Gaba, D. M., Fish, K. J., Yang, G., \& Sarnquist, F. H. (1992). Anesthesia crisis resource management training:Teaching anesthesiologists to handle critical incidents. Aviation Space and Environmental Medicine, 63 (9), 763-770.

Hunag G (2016) Procedural instruction in invasive bedside procedures: a systematic review and meta-analysis of effective teaching approaches. BMJ Qual Saf. Apr;25 (4):281-94. doi: 10.1136/bmjqs-2014-003518. Epub 2015 Nov 5

Issenberg, S. B., McGaghie, W. C., Petrusa, E. R., Lee Gordon, D., \& Scalese, R. J. (2005). Features and uses of high-fidelity medical simulations that lead to effective learning: a BEME systematic review. Medical Teacher, 27 (1), 10-28. http://doi. org/10.1080/01421590500046924

Kohn LT, Corrigan JM, Donaldson MS. (1999) To Err is Human - Building a Safer Health System. Washington: National Academy Press,.

Levine MF, Shorten G. (2016). Competency-based medical education: its time has arrived. Can J Anesth; 63: 802-6.

Makary, M. A., \& Daniel, M. (2016). Medical error - the third leading cause of death in the US. BMJ, 2139 (May), 1-5. http://doi. org/10.1136/bmj.i2139

Miller G. (1990) The assessment of clinical skills/competence/ performance. Acad Med; 65: S63-7.

Miller, R. D. (2009). Miller's Anesthesia 7th Edition. Churchill Livingston. 
Fuentes et al.

Paige, J. T., Arora, S., Fernandez, G., \& Seymour, N. (2015). Debriefing 101: Training faculty to promote learning in simulation-based training. American Journal of Surgery, 209 (1), 126-131. http://doi. org/10.1016/j.amjsurg.2014.05.034

Salas, E., Wilson, K. A., \& Lazzara, E. H. (2008). Simulation-Based Training for Patient Safety: 10 Principles That Matter. Journal of Patient Safety, 4 (4), 3-8. http://doi.org/10.1097/PTS.0b013e3181656dd6
23. Schwid, H. A., \& O'Donnell, D. (1992). Anesthesiologists'management of simulated critical incidents. Anesthesiology. http://doi. org/10.1097/00000542-199204000-00002 